\title{
PENSAMIENTO Y POLITICA UNIVERSITARIA EN AMERICA LATINA*
}

\author{
Jesús M. García del Portal**
}

\begin{abstract}
La verdadera prueba de una inteligencia superior es poder conservar simultáneamente en la cabeza dos ideas opuestas y seguir funcionando. Admitir, por ejemplo, que las cosas no tienen remedio y mantenerse sin embargo decidido a cambiarlas.
\end{abstract}

Francis Scott Fitzgerald

\section{RESUMEN}

Mientras muchos académicos y directivos, de diferentes países y organizaciones internacionales, enfocan la problemática universitaria en oposición a las condiciones del orden neoliberal predominante -aunque con matices que varían entre lo radical, lo ambiguo y lo ingenuo-otros abogan por la irremediable aplicación de medidas en adaptación al status quo. Este trabajo es un intento de resumir lo expresado anteriormente sobre la base fundamental de una investigación documental - destacando opiniones de algunos colegas expertos - así como también de las experiencias del autor en intercambios con estudiantes, profesores y directivos de la educación superior de diferentes países de la región. Además, las propias concepciones del autor sobre educación, estado e ideología en América Latina constituyen las categorías fundamentales de su crítica.

Palabras clave: Educación superior. Politica. America Latina.

Es ya un lugar común en el medio académico hablar de modernidad, de conocimiento, de neoliberalismo, privatización y restricciones financieras; de entorno y redes, de Universidad-Empresa, competitividad y acreditación, de equidad, calidad y excelencia, de pertinencia, masi-

* Artigo recebido em 26/116/2008 e aprovado em 14/03/2009.

** Jesús M. García del Portal (in memoriam) foi Doutor em Educação. Professor e pesquisador do Centro de Estudos de Aperfeiçoamento de Ensino Superior (CEPES) da Universidad de La Habana, Cuba. Professor sênior da Cátedra Unesco sobre Gestão e Docência. E-mail: cepes@cepes.cu 
ficación, diversificación, autonomía responsable y libertad académica; de "accountability" y gestión, de colaboración internacional e integración, de transformación y cambio, y, por supuesto, de globalización. El análisis de buena parte del pensamiento inherente al discurso y las políticas universitarias predominantes en nuestra región nos hace dudar en cuanto a la certeza o la posibilidad de lograr la "globalidad solidaria", el "nuevo pacto académico"; del "conocimiento como bien no escaso", del "tesoro que encierra la educación", de la equidad, la pertinencia, la disminución del "éxodo de competencias" y otras tantas invocaciones de la retórica sobre el desarrollo de la Universidad en la actualidad. Mientras muchos académicos y directivos, de diferentes países y organizaciones internacionales, enfocan la problemática universitaria en oposición a las condiciones del orden neoliberal predominante -aunque con matices que varían entre lo radical, lo ambiguo y lo ingenuo - otros abogan por la irremediable adaptación al status quo.

Este trabajo es un intento de resumir lo expresado anteriormente sobre la base fundamental de una investigación documental - destacando opiniones de algunos colegas expertos - así como también de las experiencias del autor en intercambios con estudiantes, profesores y directivos de la educación superior de diferentes países de la región, y nuestras propias concepciones sobre Educación, Estado e Ideología en América Latina, como categorías esenciales para la fundamentación de nuestra crítica.

A la interpretación de los términos anteriores por parte de algunos directivos y académicos, anuentes con las imposiciones neoliberales; radical, ambigua o ingenuamente, se opone un fuerte discurso contestatario de otros, que sin embargo aún no nos ha dotado de una alternativa realmente integradora en cuanto a guías de pensamiento y elaboración de políticas, que constituyan nuestra respuesta coherente a los cuestionamientos que se ciernen sobre la Universidad con propuestas de transformaciones y cambios de resultados cuestionables. A ello dedicamos algunas reflexiones expuestas en este documento.

Hace ya tiempo, pero sobre todo a partir de esta década, las instituciones de educación superior se han visto cuestionadas en cuanto a su pertinencia, por lo que, muy especialmente en el mundo subdesarrollado, son urgidas para aumentar su velocidad de cambio -muchas veces en direcciones no deseadas o en condiciones de incertidumbre- y en la mayor parte de los casos obligadas a seguir tendencias impuestas por un 
conjunto de factores; políticos, científico-técnicos, económicos, sociales e ideológico-culturales que se sintetizan en la llamada Globalización, que rebasa con creces, en sus dimensiones política e ideológica, su conceptualización como proceso económico, financiero y científico-técnico.

Ahora bien, al proceso anterior, acompañado en lo económico por el modelo neoliberal y de las políticas que le son inherentes, se le denomina Globalización Neoliberal y es expresión del nuevo estadío del modo de producción capitalista, continuador de la vocación internacional del capital y de un novísimo reparto del mundo - apoyadas por un notable y acelerado progreso técnico y una favorable correlación internacional de fuerzas - que trasciende zonas de influencia económica y política, estrechos marcos geográficos, sentimientos de nacionalidad e identidad cultural y "obsoletas fronteras nacionales". En esto último hay que tomar en cuenta que mientras unos poderes globalizan otros son globalizados. Los poderes globalizadores, si bien tienden a irrespetar y eliminar las fronteras de los países más débiles, mantienen, sin embargo, sus fronteras y no permiten que éstas sean irrespetadas.

La Globalización Neoliberal lleva implícita una neotransnacionalización, que apoyada en la fuerza indiscutible del capital se expresa no sólo en lo económico y financiero, con la canonización del mercado y su absoluta libertad como salomónica y universal solución, sino en lo político - desapercibida de "anacrónicos" límites fronterizos - con una "moderna" concepción del Estado; en lo social con también nuevas concepciones de identidad y en lo cultural-ideológico con el resurgimiento de la filosofía del Fin de la Historia y fundamentos que afectan decisivamente en la formación del individuo. Predominan concepciones economicistas; calidad de vida como cantidad de cosas y no reconocimiento de valor a todo lo que no tiene precio. De aquí la tendencia a convertir la educación -fuente de conocimiento y por ende del factor de producción fundamental contemporáneo- exclusivamente en un valor de cambio (PorTaL, 1995, p.21).

En cuanto al concepto de modernidad, abordado sociológicamente, destacamos que para Marx lo que está en la base de la modernidad es el surgimiento del capitalismo y de la burguesía revolucionaria (IBAÑES, inédito). Para Weber la modernidad tenia que ver con una forma especial de racionalización que solo se había dado en occidente con valor y significado universales. A partir de estas y otras conceptualizaciones del fenómeno en cuestión, nos inclinamos por entender 
el concepto de modernidad no referido a una forma cualquiera de organización social, de organización de la vida, de autopercibirse. Se refiere por sobre todas las cosas a como este proceso se percibe y se incorpora a la conciencia a partir de las consideraciones de Europa, de donde resulta que el concepto de modernidad, pasa a formar parte de las categorías que establece la cultura occidental (LóPEZ, inédito).

La modernización del mundo ha implicado que esta noción sea entendida como difusión/sedimentación de los valores y patrones socioculturales de la llamada Europa Occidental y de la América anglosajona, más concretamente de los Estados Unidos de América. Por eso, la modernización no es más que occidentalización, con sus procesos de secularización, individualización, urbanización, industrialización, mercantilización, racionalización (BELLO, 1998).

Es así que a la modernización le son inherentes categorías integrantes del núcleo duro de la Globalización y puede

"ser vista como una teoría de la cultura mundial, entendida como cultura de masas, mercado de bienes culturales, ... " que determina nuestro modo de vida, que informa la ideología dominante, y cuyos "medios de comunicación social y de producción de bienes de consumo masivo, organizados en redes mundiales nos imponen una suerte de homogeneización sociopolítica y cultural en la que la racionalidad que singulariza la civilización occidental se convierte en «el» parámetro de análisis de todas las demás formaciones sociales" (BELLo, 1998a).

Así concebidos ambos términos, la tenaza modernización-globalización es el fundamento de la nueva ideología que encubierta, edulcorada o abiertamente trata de imponérsenos como irremediable.

Una revisión del contenido implícito en el uso de otros términos inicialmente relacionados, a partir del análisis del pensamiento inherente al discurso y las políticas universitarias que tienden a imponerse en la región, nos revela que:

Las políticas de corte neoliberal sujetas a los cánones del Banco Mundial no sólo fundamentan la disminución del financiamiento estatal destinado a la educación superior para aumentar los correspondientes a los niveles primario y medio (SAMOFF, 1999), basados en las magnitudes de las tasas de retorno de las inversiones en educación para nuestros países, donde aquellas son mayores mientras menor es el nivel educacional, sino 
también una redefinición de las relaciones Universidad-Estado diferente, donde este último deja que el mercado tome su lugar para financiar la educación, pero a la vez endurece sus controles ideológicos, dando lugar, en la educación superior, a "dos dinámicas aparentemente contradictorias: el laissez faire (dejar hacer, dejar pasar) y el intervencionismo estatal (Puiggrós, 1996) " cuyo efecto combinado deriva en lo que Schugurensky (1998) denomina una universidad heterónoma (WEBER, 1947).

La privatización de la educación es otro de los principios fundamentales para el desarrollo de la educación establecidos por el Banco Mundial. Este anunciado Prometeo para la Universidad tiene también sus peligros. Si bien es cierto que algunas de las más prestigiosas universidades de América Latina son privadas, muchas de ellas de la Iglesia Católica, también lo es que se incrementa el número de las instituciones privadas que están entre aquellas de "absorción de demanda" que ofrecen certificados de calidad cuestionable y de incierta garantía en el mercado laboral (AltBACH, 1998, p.9).

Al respecto de la privatización y especialmente en cuanto a la investigación en las universidades latinoamericanas, Levy pone de manifiesto que "un audaz nuevo mundo de organizaciones sin fines de lucro han aparecido en toda América Latina", los denominados Centros de Investigación Privados (CIP) que son una parte crucial del repentino y vigoroso sector sin fines de lucro latinoamericano. En tal sentido afirma que

los CIP han desplazado a las universidades públicas como líderes en la producción y diseminación de ciencia social e investigación sobre políticas (es decir, "investigación social"), dando como causas de ello el que las universidades latinoamericanas han sido generalmente incapaces de poder integrar enseñanza e investigación, al mantener la prioridad de la formación profesionalizante, el estilo predominante de enseñanza como antitético con el desarrollo de la investigación y el hecho de que muchos de los profesores a tiempo completo hacen poca investigación (LEVY, 1996, p.1, 71-72).

Igualmente considera como un importante factor, que lesiona la investigación universitaria, a la presencia del activismo político en su interior.

Por otra parte, la primera Cumbre Iberoamericana de Rectores de Universidades Estatales constató que 
la universidad pública afronta en la actualidad la imposición del modelo neoliberal y de la economía de mercado, que fija como meta de la educación superior, formar para el mercado, y que tiende a privatizar toda actividad. Secuela de esto es la especialización con criterios de rentabilidad profesional, especialización sin formación cultural [...] Otro peligro es el conformismo social y político. Lo que expresa el tono de la vida actual y de la universitaria en particular, pues sugiere que apuntamos a una comunidad de sobrevivencia más que de realización personal (I Cirue, 1999).

Realmente esta situación conforma una "tendencia que se extiende en universidades que se adaptan pasivamente a las demandas del mercado laboral, con el respaldo de la ideología neoliberal" (Pons, 1995, p.144) y cuya apología se constituye en una

viscosa doctrina que insensiblemente envuelve a todo razonamiento rebelde, $[\ldots]$. el pensamiento único [...] la traducción en términos ideológicos, pretendidamente universales, de los intereses de un conjunto de fuerzas económicas, en particular las del capital internacional" (RAMONET, 1995).

La pertinencia, tan de moda en el discurso universitario actual, muy a menudo es concebida, con ingenuidad o aparente desconocimiento, tal cual conviene a las políticas neoliberales, es decir como una categoría determinada fundamentalmente por su significación económica. "La pertinencia tendrá que ser demostrada, no una vez sino continuamente. Los imperativos económicos barrerán con todo lo que se les oponga y si las universidades no se adaptan, se las dejará de lado" (GiBbons, 1998, p.2). Simultáneamente

han surgido documentos y declaraciones que abogan por la necesidad de un "nuevo contrato social», un "nuevo pacto" entre las instituciones de educación superior y los gobiernos, lugar común reiteradamente manejado en esos mismos términos, prácticamente en todos los documentos del Banco Mundial (BM) y del BID referidos a la educación superior" (Pons, 1998, p.141).

De aquí que coincida con la apreciación de que es "esencial tener claro cuál es el origen y la intención de determinadas propuestas "transformadoras" que surgen como hongos y de manera simultánea en distintos países" (op. cit.p.111). 
No es posible desconocer tampoco que, la visión del Banco sobre la educación tiene la capacidad de penetrar en los ámbitos de la toma de decisiones en la región (Morales-Gómez, p.13-17), es decir que su poder se ejerce también, de manera decisiva, sobre el pensamiento de los gobernantes y por tanto en las políticas ejercidas por ellos, en virtud de que su Agenda Político-Ideológica esta sustentada en la mas poderosa combinación de recursos financieros, experiencia técnica e influencia política dentro de las organizaciones internacionales, constituyendo hoy realmente lo que Samoff (1991) denominó un "complejo financiero-intelectual".

Como fortaleza en el desfavorable panorama universitario regional creo en la existencia de una masa crítica de profesores, opuestos al deterioro de la Universidad ante las evidentes amenazas, ataques y cercos presupuestarios gubernamentales a la misma, que luchan por la elaboración de respuestas constructivas, así como del ejercicio autocrítico ante la necesidad de profundizar en el criterio de que "muchos de los problemas que enfrentan las universidades son autoinfligidos y surgen de su interior" (Vessuri, 1996, p.106). Por tanto, es preciso consagrar una mayor proporción de nuestros pensamientos y esfuerzos a la búsqueda de acciones alternativas para vencer el acoso hacia el ocaso de la universidad latinoamericana. Una de ellas, me atrevo a asegurarlo, es el logro de la integración universitaria latinoamericana. Aunque en lo inmediato, y tal como opina Ares Pons,

creemos que debe profundizarse el examen de las posibilidades de concretar las aspiraciones planteadas -universalización de la enseñanza terciaria y otras- a la luz de las realidades imperantes en la sociedad neoliberal que padecemos -mercado de trabajo deprimido, ofensiva contra la enseñanza pública, etc. Tengo la impresión de que estamos mirando los problemas desde la perspectiva muy acotada del mundo de la educación, aislándonos del ámbito mayor de las luchas sociales, sin cuya contribución es impensable que aquellas aspiraciones puedan concretarse ... Creo que está haciendo mucha falta un enfoque realista de estos temas (Pons, 2002).

Continuando con el enfoque de la problemática objeto de análisis al caso de nuestra región, un vistazo al entorno socioeconómico, político e ideológico latinoamericano y caribeño nos hace dudar en cuanto a la certeza o la posibilidad de lograr la "globalidad solidaria", el "nuevo 
pacto académico", el "conocimiento como bien no escaso", el "aprender a ser, a conocer, a hacer y a vivir juntos", la equidad, la pertinencia, la disminución del "éxodo de competencias" y otras tantas invocaciones del discurso sobre el desarrollo de la Universidad en la actualidad. Vale la pena repasar algunas cifras, hechos y apreciaciones.

América Latina y el Caribe, tiene una extensión territorial de 20,5 millones de kilómetros cuadrados y una población de 500 millones de habitantes, es decir que con respecto al entorno mundial ocupamos el $15,16 \%$ de la superficie terrestre del planeta y el 8,33\% de la población total. Más de mil millones de habitantes en el mundo viven en la pobreza; de ellos 250 millones son latinoamericanos y caribeños y de estos casi la mitad en nivel crítico. Somos la región con la más injusta distribución de la riqueza en el mundo (el ingreso del $20 \%$ de la población más rica supera de 10 a 15 veces el del $20 \%$ de la población más pobre). El número total estimado de analfabetos adultos es de casi 900 millones, de ellos 42,9 en nuestra región, el 55\% de los cuales son mujeres. América Latina invierte menos en educación que todas las otras regiones del mundo (REIMERS, 1994).

Otro triste presagio, esta vez de Naciones Unidas, se refiere al crecimiento sin empleo (PNUD, 1993). Mientras que el PIB mundial crecerá 2,05 veces entre 1975 y el 2000, el empleo solo lo hará en 1,47 veces. En el caso de América Latina las cifras respectivas son 1,91 y 1,48. Esto último resulta agravado aún más en esta década en nuestra región, al considerar que mientras la fuerza de trabajo se incrementará entre 1990 y el 2000 en 1,27 veces el empleo solo crecerá 1,14 veces.

A finales de la década pasada, en un análisis resumen efectuado por la CEPAL sobre la disminución del desarrollo económico de la región durante esos años, se expresaron sentimientos entremezclados y simultáneos de melancolía, amargura y esperanza (RodRíGuEz, 1998, p.4). En aquellos tiempos, algunos académicos coincidieron en la creencia de que a pesar de los graves problemas de la enseñanza superior, las potencialidades universitarias podrían contribuir de manera importante para lograr que el decenio perdido de los años 80 quedase sólo como un período de transición, como un amargo conjunto de lecciones y no como un rasgo permanente de las sociedades latinoamericanas. Lamentablemente, no fue posible que la que algunos más optimistas llamaron la década del "aprendizaje doloroso" fuese tal, a no ser porque aprendimos que el dolor continuaría. 
Es coherente con lo anterior el que la propia CEPAL, en su tesis para el desarrollo de América Latina y el Caribe en esta década planteara la

eliminación del profundo desfase entre el sistema educacional tradicional y los requerimientos de la sociedad que se perfila para el siglo XXI; accesibilidad a los códigos de la modernidad; alcance de mayores niveles de representatividad y participación en el ejercicio de la ciudadanía; acercamiento entre el sistema educacional, el mundo de las comunicaciones y la esfera del trabajo; eliminación del alto riesgo de desintegración y de multiplicación de las desigualdades, que podría conducir a una creciente diferenciación entre minorías formadas para manejar el futuro y mayorías vinculadas al pasado o excluidas del dinamismo de la modernidad (CEPAL, 1992, p.102).

En el ámbito sociopolítico son premonitorias las opiniones, por solo mencionar algunas, del conocido sociólogo francés Alain Touraine cuando señaló en algunos de sus trabajos que es característico de las sociedades latinoamericanas la disociación entre el pensamiento y la acción (Touraine, 1976, p.58 e ss.), debidas a la separación entre lo económico y lo social, entre la ideología y la política, que imponen las clases dominantes. Juan Carlos Tedesco, más recientemente, al enfocar la problemática de la Gestión Pública en cuanto al trazado de estrategias de Desarrollo y Educación en América Latina señala la distancia entre retórica y resultados.

Por su parte Adriana Puiggrós (1998) avanza desde posiciones conceptuales hacia la fundamentación de las razones que han favorecido el afianzamiento de la racionalidad de las reformas neoliberales en política educativa, cuando afirma que ello ha sido facilitado por

la insuficiencia de una izquierda actualizada, las críticas demasiado reactivas y carentes de alternativas consistentes", así como que tal situación es "una consecuencia más de las insuficiencias que caracterizan a los sectores progresistas en este fin de siglo (p.46).

Estas aseveraciones, y su vinculación en nuestro análisis del impacto ideológico en la región de la desaparición del socialismo en Europa y la consolidación del neoliberalismo, nos hace recordar los polémicos planteamientos de Petras (1990) al hablar del "repliegue de los intelectuales" desde el marxismo al "realismo", al acomodamiento y el utilitarismo, y también a la "aclimatación" intelectual a las nuevas 
fuentes de poder; así como la fuerte réplica de Vilas (1990) en su explicación del fenómeno en América Latina, sus consideraciones sobre los intelectuales latinoamericanos que denominó "aplaudidores acríticos" y su reconocimiento preocupado de que "los intelectuales críticos [...] son fruta poco frecuente en el jardín de las ciencias sociales críticas latinoamericanas"

Otras críticas fuertes en cuanto al significado y profundidad de los resultados de nuestro trabajo académico, tanto en lo teórico como en lo práctico, en las últimas décadas, las tenemos en algunas expresiones de dos reconocidos investigadores estadounidenses de la Universidad en América Latina, como Robert Arnove (1967) quien afirmó en 1967: "La literatura sobre la universidad en América Latina es fundamentalmente de naturaleza polémica y especulativa, exenta en general de base empírica y de significación teórica" y más recientemente Daniel Levy (1995, p.51), cuando al pronunciarse sobre lo dicho por Arnove en aquel entonces expresa: "aunque algunos trabajos han mejorado esta situación, la afirmación de Arnove todavía mantiene su peso".

Independiente de la certeza que mantengan actualmente las aseveraciones anteriores, ellas ponen en evidencia otro importante aspecto crítico, digno de tomar en consideración, consistente en el hecho de que una parte apreciable de la literatura que identifica, explica y propone soluciones a los problemas de la educación en nuestra región, proviene de investigadores y académicos de los Estados Unidos de América, e incluso de latinos formados en las universidades de aquel país. Ello motiva la reflexión de que, sin pretender disminuir en algo dichos estudios por colegas y en muchos casos amigos de otros países, se impone la necesidad de potenciar las capacidades y posibilidades endógenas en tal sentido, aunque es sabido que si ello no ha podido ser así desde antes se debe también en buena medida a razones de carácter objetivo incluidas en las refutaciones de Vilas a Petras, cuando sostiene que los intelectuales latinoamericanos pierden mucho más que su fuente principal de ingresos al ser echados de sus universidades, donde por demás los salarios no son ni lejanamente comparables a los que se pagan en las instituciones estadounidenses, amén de las grandes diferencias en las condiciones de trabajo académico entre ambos medios.

No debemos pasar por alto que en cuanto a la generación de cultura científica y nuevo conocimiento no solo somos pobres, sino que también condenados por algunos pronósticos a una eterna dependencia externa. 
Actualmente, (...), desde diversos ámbitos se postula claramente la necesidad de optar por una política dirigida a formar para el uso adecuado del conocimiento disponible, antes que para su producción" (...) "Optar por formar para el uso del conocimiento se presenta como una opción mas equitativa que formar para la producción de conocimientos" (TEDESCO, s.d.).

Luego de estas breves citas sobre el complejo "estado del arte" de la Universidad en América Latina, entiendo que el reto al que nos enfrentamos tiene dimensiones, en lo político e ideológico, que trascienden con creces el enfoque solo desde los ámbitos económico, científico-técnico y puramente académico. Cada vez gana más fuerza la tendencia a buscar soluciones "exclusivamente técnicas a problemas que son esencialmente de naturaleza política". (VERA, 1994).

Por todo ello considero que los investigadores sobre la Universidad debemos priorizar el encuentro en común de estrategias mejores, mas justas, de desarrollo universitario, ante los retos del entorno más complejo que haya tenido nunca nuestra Alma Mater, saturada ya de ejercicios discursivos, a veces fines en sí mismo, que no rebasan el ser nuevas versiones de lo ya conocido, y tienden a la copia de recetas y a hacernos eco de elaboraciones y voces foráneas. Es cierto que estas "adaptaciones" se nos imponen mediante fuerzas muy presionantes, en lo económico, político y también intelectual (CorAGGIO, 1988), pero igualmente lo es que parecemos imposibilitados de que la masa crítica contestataria pueda hacer oír sus voces con propuestas alternativas de intelectuales comprometidos con la esperanza y convencidos de que sí hay soluciones y de que somos nosotros mismos quienes debemos y podemos encontrarlas.

Aspiro a expresar, sin excesos de hipercriticismo, un avisoramiento alertador de que lo dicho al inicio tiende a sumirnos en un marasmo de términos, algunos de los que ya incorporamos a nuestras nomenclaturas sin percatarnos de su verdadero y peligroso sesgo economicista, pretendidamente despolitizado y desideologizado, que nos hunde nuevamente en copias acríticas y en el desarme conceptual de nuestras inteligencias, cayendo en falacias, ingenuidades y en la pérdida cada vez mayor de nuestro necesario ethos académico. Aún más, creo que es preciso poner en tela de juicio las elusivas y candorosas interpretaciones del Estado, que intentan presentarlo como un ente ideal neutro en lo político e ide- 
ológico, conciliador de los intereses entre opulentos y desposeídos, y no como un aparato, según Althusser (1989), que expresa la voluntad de la clase dominante para el ejercicio del poder y la imposición de su ideología mediante un sistema de instituciones, una de ellas la escuela. Igualmente, la pretendida verdad absoluta de la necesidad de un Estado pequeño, enajenado de anacrónicas responsabilidades - como la salud y la educación públicas - y desideologizado, inherentes al modelo neoliberal, realmente portador de una ideología hegemónica anuente con los razonamientos de Fukuyama (1992). Tal vez podríamos coincidir con Norbert Lechner en su afirmación de que "la cuestión no es menos Estado o más Estado, sino otro Estado" (apud BERNHEIM, 1996, p.13). Pero: ¿cuál es ese otro Estado?

No se trata de desconocer voluntaristamente realidades - aunque sean indeseables - ni las dimensiones económica y científico-técnica del progreso del mundo actual, mucho menos de desechar aportes de ciencias como la Economía, la Gestión, la Computación u otras. Tampoco de ignorar problemas autoinfligidos, negándonos a ultranza a considerar la necesidad de analizar posibles adecuaciones de la Universidad Latinoamericana contemporánea luego de casi medio milenio de existencia, aunque como ejercicio de Reforma generado al interior de la misma considerando el contexto externo y no exclusivamente como adaptación impuesta por este último como condición sine qua non.

Se trata, ante todo, de conocer esencialmente las contradicciones de ese entorno globalizado y muchas veces excesivamente edulcorado que se tiende a interpretar y aceptar ingenuamente, soslayando y también resignándonos a su apellido neoliberal. De aquí que, por ejemplo, no se percaten algunos que el conocimiento siempre ha tenido valor y que lo que hoy está presente en el mismo como "nuevo" es exclusivamente su nuevo y exacerbado valor económico, que a su vez lo ha convertido en el componente del poder (armas, riqueza y conocimiento) peor distribuido en el mundo (Toffler, 1990, p.545); así como que la en muchos casos deformada interpretación de los términos aludidos al inicio informan una retórica que lejos de iluminar la elaboración endógena de justas políticas y estrategias alternativas, hacen caer a muchos en discursos anuentes en definitiva con el status quo mercantilista que se nos impone, como un agujero negro cuya enorme fuerza de atracción depende de la variable valor de cambio, aún de lo que no tiene precio. 
Que los criterios de equidad, pertinencia, acreditación, calidad, excelencia y colaboración internacional estén en función de nuestras identidades, contextos específicos y proyectos sociales, sin que ello implique el desconocimiento de patrones y normas internacionales de obligatoria consideración. Que no se hable tanto de sociedad, conocimiento y democracia sin plantearse seriamente que la sociedad del conocimiento no será posible sin la democratización del conocimiento, debiendo encauzarse las acciones hacia el logro de garantías para la educación como políticas de Estado y no de gobiernos, evitando que las estrategias educacionales queden sometidas a los cambios relativamente a corto plazo de las administraciones e incluso de ministros y funcionarios gubernamentales, en búsqueda de garantías reales para que el tesoro que encierra la educación pase a ser patrimonio cultural de la Humanidad. !Que la llave del candado del tesoro sea un bien común!

Que nuestras retóricas iluminen la elaboración y puesta en práctica de alternativas factibles eliminando falacias y frases consoladoras, tales como que el conocimiento no es un bien escaso, que somos el mayor potencial científico de cada país de la región, así como la inaceptable y simplista reducción de la causa de la "crisis universitaria" a las limitaciones financieras, con ignorancia o desconocimiento de que dicha "crisis"- sin ocultar importantes problemas al interior de la Universidad es primero que todo consecuencia de la verdadera crisis del sistema socioeconómico y político vigente, de ese Estado y de esa ideología incapaces de dar respuesta a las necesidades de las mayorías.

Si bien parece que el siglo culminará con democracias formales en prácticamente todos los países de América Latina, la debilidad de éstas es evidente" [...] lo que falta no es el dinero, sino la voluntad y la capacidad de orientar el accionar conjunto hacia la reducción de las desigualdades existentes, de impulsar el desarrollo humano sostenible, de perfeccionar la democracia y construir la paz, con el fin de mejorar la calidad de vida de las mayorías (YARZABAL, 1999, p.10 e 17).

Que no se nos convoque más, tampoco, a la elaboración de nuevas teorías y prácticas, al reto de hacer una reingeniería social que redefina la educación para que este a tono con las nuevas tendencias mundiales, en otras palabras, para que se ponga en correspondencia con el sistema que ha originado la crisis. 
En fin, que todo sea un problema de cambio -otro término fetichizado y puesto de moda- pero del cambio de todo para que en definitiva todo lo esencial permanezca sin cambiar.

\section{CONCLUSIONES}

Aunque en el párrafo final del resumen quisimos expresar que nuestro trabajo es un intento de síntesis de categorías y concepciones, para fundamentar nuestra crítica al estado del arte de la problemática a que alude el título, así como para fundamentar los criterios del autor al respecto, podemos precisar aquí la idea central que nos inspiró en la elaboración del trabajo y que tratamos de exponer a través del mismo.

Se trata en esencia de defender la idea de la necesidad y responsabilidad que la Universidad y los universitarios tienen de generar un pensamiento autónomo y contribuir a la realización de las transformaciones que requiere el desarrollo del país, en sus dimensiones económica, social y cultural e ideológica, que nos dote con los fundamentos requeridos para la elaboración de alternativas viables para enfrentar la Globalización Neoliberal.

En lo universitario, el conocimiento de nuestro medio académico es expresivo, en gran medida, de la existencia de pugnas entre las instituciones y los organismos nacionales de coordinación, entre las mismas instituciones, y mas aún en el seno de cada una de ellas; como consecuencia de sus contradicciones internas, de políticas partidistas, y de tergiversadas manifestaciones de autonomía y cogobierno, entre otras. Es prácticamente inexistente, en el discurso universitario, la problemática de los maestros y la vinculación Escuela-Universidad, y en cuanto a la sociedad en general es muy débil aún el desarrollo de la Extensión Universitaria en todas sus posibilidades, lo que contribuiría tanto al desarrollo socioeconómico del país como al incremento del prestigio político de la Universidad en su condición de institución social.

Ahora bien, para la eliminación de los males que nos aquejan, son imprescindibles, como fundamentos del reto a que nos enfrentamos, como condición sine qua non la Integración Universitaria que necesita nuestra Región en general y nuestra Universidad en particular. Si no logramos que dicha Integración abarque desde el interior mismo de cada universidad hasta el de los países latinoamericanos, será de todo punto de vista impracticable llevar a cabo las transformaciones en el pensamiento 
y las políticas de desarrollo de nuestra educación superior. Toma aquí nueva vigencia, ante la presencia del sistema mundial hegemónico más agresivo que hayamos padecido, el sueño de nuestros próceres, encabezado por el Libertador Simón Bolívar, quien expresara hace ya casi dos siglos: "Los pueblos marchan, hacia el término de su grandeza, con el mismo paso con q camina la educación".

\begin{abstract}
While many academics and boards of directors from different countries and international organizations focus the problem of universities in opposition to the conditions of the prevailing neoliberal order -though with nuances varying from the radical, the ambiguous to the ingenuous- others favor the irremediable adaptation of the status quo. Based on documentary research, this work is an attempt to summarize this position highlighting opinions of experts, experiences of the author in exchanges with students, professors and higher education authorities of the different countries in the region. Moreover, the author's own conceptions about education, state and ideology in Latin America constitute the fundamental categories to the author's perspective.
\end{abstract}

Keywords: higher education, policy, Latin America.

\title{
RESUMO
}

Enquanto muitos acadêmicos e dirigentes, de diferentes países e organizações internacionais, enfocam a problemática universitária em oposição às condições da ordem neoliberal predominante - ainda que com matizes que variam entre o radical, o ambíguo e o ingênuo, outros defendem a irremediável aplicação de medidas para a adaptação ao status quo. Este trabalho é uma tentativa de resumir o que foi expresso anteriormente sobre a base fundamental de uma investigação documental - destacando opiniões de alguns colegas especialistas - assim como, também, das experiências do autor em intercâmbios com estudantes, professores e dirigentes da educação superior de diferentes países da região. Além disso, as próprias concepções do autor sobre educação, estado e ideologia na América Latina, constituem as categorias fundamentais de sua crítica.

Palavras-chave: educação superior. Política. América Latina 


\section{REFERENNCIAS}

ALTBACH, P. G. The Anatomy of Private Higher Education, International Higher Education, The Boston College Center for International Higher Education, Number 12, Summer 1998, p. 9.

ALTHUSSER, L. Ideología y aparatos ideológicos del Estado (Notas para una investigación). In: La Filosofía como Arma de la Revolución, 18va.. Edición, Siglo XXI, México, 1989.

ARNOVE R. A Survey of Literature on Latin American Universities. In: Latin American Review 3, p. 45-53, s.1., s. ed.,1967.

BELLO, M. E. La Educación en Iberoamérica, Organización de Estados Iberoamericanos para la Educación, la Ciencia y la Cultura, 1998.

. La gobalizatión como fenómeno cultural. Organización de Estados Iberoamericanos para la Educación, la Ciencia y la Cultura, 1998a.

BERNHEIM, C. T. Conferencia introductoria a la "Conferencia regional sobre políticas y estrategias para la transformación de la educación superior en América Latina y el Caribe". La Habana, Cuba, 18 al 22 de noviembre de 1996, Caracas: CRESALC/Unesco, 1996.

CEPAL, "Educación y Conocimiento: Eje de la Transformación Productiva con Equidad", Santiago de Chile: CEPAL-OREALC, 1992,

CORAGGIO, J. L. "Investigación Educativa y Decisión Política. El caso del Banco Mundial en América Latina. Perfiles Educativos, CESU-UNAM. Volumen XX, Números 79-80, 1998.

FUKUYAMA, F. El Fin de la Historia y el último hombre, Barcelona: Editorial Planeta, 1992.

GIBBONS, M. Pertinencia de la educación superior en el siglo XXI" Education. s.l.The World Bank, 1998.

I CIRUE, Primera Cumbre Iberoamericana de Rectores de Universidades Estatales. Acuerdo de Santiago. Reunión de Chile. Santiago: Universidad de Santiago de Chile, agosto 1999.

IBAÑEZ. Modernidad, razón e identidad en América Latina. Santiago de Chile: Editorial Andrés Bello, 1996.

LEVY D. C. Building the Third Sector. Latin America's Private Research Centers and Nonprofit Development. Pittsburgh: University of Pittsburgh Press, 1996.

. La educación superior y el Estado en Latinoamérica. Desafíos privados al predominio público. Ciudad de Mexico: Grupo Editorial Miguel Angel Porrúa, 1995. 
LINDO, A. P. Políticas del conocimiento, educación superior y desarrollo. Buenos Aires: Editorial Biblos, 1998.

LÓPEZ L. B. Modernidad, Modernización y Aldea Global: La Encrucijada de la Universidad Latinoamericana. Tema de examen para optar por el Grado Científico de Doctor en Ciencias de la Educación. La Habana: CEPES-Universidad de La Habana (inédito);

MORALES-GÓMEZ D. A.; TORRES, C. A. (Editors). Research, Knowledge, Power, and Policy. In: . Education, Policy, and Social Change". USA: Praeger Publishers, 1992.

PETRAS, J. Los intelectuales en retirada. Nueva Sociedad, Caracas, Venezuela, n. 107, mayo-junio/1990. p.92-120.

PNUD. Informe sobre Desarrollo Humano. Madrid: Ediciones Mundi-Prensa, 1997

. Informe sobre Desarrollo Humano, New York, s.ed.,1993.

PONS, J. A. La Universidad Latinoamericana en el ojo de la tormenta. In: Universidad: ¿Anarquía Organizada? Uruguay: Librería de la Facultad de Humanidades y Ciencias de la Educación, Universidad de la República, 1995, p. 144.

. Córdoba 2000, Educación Superior y Sociedad. Caracas: IESALC/ Unesco, v. 9 n. 1, p.129-144.

. Presente y futuro de la universidad latinoamericana, Educación Superior y Sociedad, Caracas: IESALC/Unesco, v. 7 n. 1, 1996, p. 109-126.

PORTAL, J. M. G. Seis ensayos sobre: Educación y Transformación Social: Apreciaciones". Facultad de Ciencias Sociales. Escuela Académica de Sociología. Lima: Universidad Nacional Mayor de San Marcos, Octubre,1995.

PUIGGRÓS A. World Bank Education Policy: Market Liberalism Meets Ideological Conservatism, NACLA Report on the Americas,s.1., s.ed. v. 29, n. 6, mayo-junio de 1996.

. Educación Neoliberal y Alternativas. In: Alcántara, A.; Pozas, R. e Torres C. A. (Coordinadores). Educación, Democracia y Desarrollo en el Fin de Siglo. México: Siglo Veintiuno Editores, 1998.

RAMONET, I. El pensamiento único. Le Monde Diplomatique, París, enero 1995.

REIMERS, F. Education, Equity and Economic Competitivness in the Americas: An Inter-American Dialogue - OEA Project", s.l., s. ed. 1994.

RODRÍGUEZ, C. R. Discurso en ocasión del aniversario 40 de la CEPAL. Periódico GRANMA, La Habana, miércoles 27 de abril de 1988. 
SAMOFF, J. Cuáles son las prioridades y estrategias para la educación? ” Revista Cubana de Educación Superior, v. XIX, n. 1, p. 3-31 y n. 2, p. 3-25, 1999.

. Research, Knowledge, and Policy Assistance to African Education: The Financial-Intellectual Complex. Paper presented at the Fifteenth World Congress of International Political Science Association, Buenos Aires, 1991.

SCHUGURENSKY. D. La Reestructuración de la Educación Superior en la Era de la Globalización: ¿Hacia un Modelo Heterónomo?”, In: ALCÁNTARA, A.; POZAS, R. y TORRES, C.A. (Coord.), Educación, Democracia y Desarrollo en el Fin de Siglo, México: Siglo Veintiuno Editores, 1998.

TEDESCO, J. C. Educación y Sociedad en América Latina: algunos cambios conceptuales y políticos. Fermentum, Ano 2, n. Especial 6 y 7, Mérida, Venezuela: ULA, s.d.

. Estrategias de Desarrollo y Educación: El Desafío de la Gestión Pública. Reforma y Utopía, s.1., n. 4, abr-jun. 1991.

TOFFLER, A. El Cambio de Poder. España: Editorial Plaza \& Janes, 1990.

TOURAINE, A. Les sociétés dépendantes. París: Duculot, 1976.

VERA, E. A. Del Discurso de la Pobreza a la Pobreza del Discurso. In: Pobreza y Educación en América Latina. Ponencia. Buenos Aires: Curso Regional sobre Gerencia Social, 23 al 27 de mayo de 1994.

VESSURI, H. Pertinencia de la Educación Superior Latinoamericana a Finales del Siglo XX, Nueva Sociedad n, 146, Nov.Dic. 1996.

VILAS, Carlos M., Sobre cierta interPetrasción de la intelecutalidad latinoamericana. Nueva Sociedad, No 107, mayo-junio 1990. Caracas, Venezuela, p.21-130.

WEBER, M. The Theory of Social and Economic Organization, Nueva York: Oxford University Press, 1947.

YARZABAL, L. Consenso para el Cambio en la Educación Superior, IESALC/ Unesco, p. 10-17, Caracas, s. ed. 1999. 